\title{
HYSTERESIS-BASED SWITCHING CONTROL OF STOCHASTIC LINEAR SYSTEMS
}

\author{
Maria Prandini*, João P. Hespanha**, M.C. Campi $^{* * *}$ \\ *Politecnico di Milano, Italy - prandini@elet.polimi.it \\ ** University of California, Santa Barbara, U.S.A. - hespanha@ece. ucsb.edu \\ ***Università degli Studi di Brescia, Italy - campi@ing.unibs. it
}

Keywords: adaptive control; switching systems; $L^{2}$-stability; stochastic hybrid systems; uncertain systems.

\begin{abstract}
We study hysteresis-based switching control for a class of discrete-time stochastic linear systems.

We take as given a family of candidate controllers that is sufficiently rich so as to include at least one controller suitable for each admissible process model. The controller operating in closed-loop with the system is replaced as soon as the measured data is significantly incompatible with the corresponding process model. In practice, this happens when the value taken by a least-squares identification cost exceeds its minimum (over all admissible models) by a certain hysteresis factor. The controller is then replaced by the one tuned to the best-fit model. We show that the proposed switching control system is stable for every value of the hysteresis factor, and that this is ensured despite of the presence of possibly unbounded noise.
\end{abstract}

\section{Introduction}

We study the problem of controlling a linear system in presence of large modeling uncertainty.

A possible way to address this issue is given by the so-called switching control approach to adaptive control originally introduced in [1] and further developed in, e.g., [2]-[9]. A switching control scheme is typically composed of an inner loop where a controller is connected in closed-loop with the system, and an outer loop where a supervisor decides -based on the inputoutput data- which controller to place in feedback with the system and when to switch to a different one.

The controller selection is typically an "estimation-based" procedure. Precisely, a parameterized model class is considered, and a candidate controller is associated with each admissible model such that it stabilizes the model when placed in feedback with it. The controller chosen to be placed in feedback with the system is the one that is associated with the best estimated model according to some identification cost. The switching times are chosen so as to avoid that switching is too fast with respect to the system settling time, thus causing instability.

In the hysteresis-based switching logic (see, e.g., [9]), slow switching is obtained by changing controller only at those times $t$ when the collected data reveal that the model used to select the currently operating controller is significantly worse than the best estimated model. More precisely, the controller currently in the loop is replaced if the value of the identification cost for the associated model exceeds the minimum by some hysteresis factor. An alternative to this logic is the dwell time switching logic, where the switching rate is slowed down by making a dwell time elapse between consecutive switching times (see, e.g., $[3,4,10,11]$ ), either by fixing it before implementing the switching controller $([3,4])$, or by selecting it on-line at each switching time $([10,11])$. The stability analysis of a dwell time switching control scheme is much easier. However, bad transients may occur because the dwell time is predetermined and may need to be large, whereas in the hysteresis switching approach the controller is replaced as soon as data show that it is not appropriate for the system.

In this paper, we study the hysteresis-based switching control of a class of stochastic discrete-time linear systems affected by possibly unbounded noise. We show that for this class of systems, when the least squares cost is used as identification criterion in the hysteresis switching scheme, stability of the adaptive control system is guaranteed.

This stability result is a nontrivial extension to stochastic systems of the results that can be found in the literature on hysteresis switching, which are restricted to a deterministic setting. In our setting, the hysteresis factor can be arbitrarily chosen, which simplifies the hysteresis-based switching logic design and allows one to make the response time of the adaptation mechanism as small as desired. This is proved in Section 4 and illustrated in the simulation example presented in Section 5.

The rest of the paper is organized as follows. In Section 2, we precisely formulate the problem by describing the class of stochastic systems we deal with, and the family of candidate controllers among which switching is performed. The supervisory control architecture is then described in details in Section 3. The hysteresis-based switching control scheme is analyzed in Section 4. A simulation example is given in Section 5. Concluding remarks are given is in Section 6.

\section{Statement of the problem}

We consider systems in input-output form described by:

$$
\mathcal{A}\left(\vartheta^{\circ} ; q^{-1}\right) y_{t+1}=\mathcal{B}\left(\vartheta^{\circ} ; q^{-1}\right) u_{t}+w_{t+1},
$$

where $\mathcal{A}\left(\vartheta^{\circ} ; q^{-1}\right)=1-\sum_{i=1}^{n_{s}} a_{i}^{\circ} q^{-i}$ and $\mathcal{B}\left(\vartheta^{\circ} ; q^{-1}\right)=$ $\sum_{i=1}^{m_{s}} b_{i}^{\circ} q^{-i+1}$ are polynomials in the unit-delay operator $q^{-1}$ and $\vartheta^{\circ}=\left[\begin{array}{llll}a_{1}^{\circ} & a_{2}^{\circ} \ldots a_{n_{s}}^{\circ} & b_{1}^{\circ} \ldots b_{m_{s}}^{\circ}\end{array}\right]^{T}$ is the system param- 
eter vector. We denote by $s_{s}$ the order of the system, i.e., $s_{s}:=\max \left\{n_{s}, m_{s}\right\}$.

The signal $w_{t}$ is a stochastic disturbance for which the following assumption holds.

Assumption 1. $\left\{w_{t}\right\}$ is a martingale difference sequence with respect to a filtration $\left\{\mathcal{F}_{t}\right\}$, satisfying: i) $\sup _{t} E\left[w_{t}^{2} / \mathcal{F}_{t-1}\right]<$ $\infty$ and ii) $\lim _{N \rightarrow \infty} \frac{1}{N} \sum_{t=0}^{N-1} w_{t}^{2}=\sigma^{2}>0$, almost surely (a.s.).

Note that Assumption 1 is satisfied, for example, when $\left\{w_{t}\right\}$ is an i.i.d. Gaussian sequence with zero mean and variance $\sigma^{2}$, but it includes many other situations. Moreover, differently from what is typically done in the switching control literature ([3, 4, 6, 7, 12]), we allow $w$ to be unbounded.

We consider the case when some coarse a-priori knowledge on $\vartheta^{\circ}$ is available. In particular, we assume that:

Assumption 2. $\vartheta^{\circ} \in \Theta$ with $\Theta \subset \Re^{n_{s}+m_{s}}$ either composed by a finite number of points or a compact set.

The admissible models set is the set of systems of the form (1) with $\vartheta^{\circ}$ replaced by $\vartheta, \vartheta \in \Theta$. Note that each model is linearly parameterized on $\vartheta \in \Theta$, since it can be rewritten in a regression-like form:

$$
y_{t+1}=\varphi_{t}^{T} \vartheta+w_{t+1},
$$

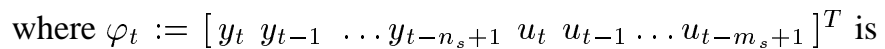
the regression vector.

In general, no single linear time invariant controller is able to stabilize all the systems in the admissible model class. We then consider a family of candidate linear time invariant controllers and introduce a supervisor that decides on-line which candidate controller is suitable for the system.

For the sake of simplicity in the implementation of controller and supervisor, we assume that the candidate controllers family is finite. Specifically, the parameter set $\Theta$ is partitioned into $m$ compact sets $\Theta_{k}, k \in \mathcal{K}:=\{1, \ldots, m\}$, such that, for each $k \in \mathcal{K}$, the models in the set $\Theta_{k}$ are robustly stabilized by the controller

$$
\mathcal{R}\left(k ; q^{-1}\right) u_{t}=\mathcal{S}\left(k ; q^{-1}\right) y_{t},
$$

where $\mathcal{R}\left(k ; q^{-1}\right)=1-\sum_{i=1}^{m_{c}} r_{i}(k) q^{-i}$ and $\mathcal{S}\left(k ; q^{-1}\right)=$ $\sum_{i=0}^{n_{c}} s_{i}(k) q^{-i}$, with order $s_{c}=\max \left\{n_{c}, m_{c}\right\}$. To be precise:

Assumption 3. For each $k \in \mathcal{K}$, the roots of the characteristic polynomial $q^{s_{s}+s_{c}}\left\{\mathcal{A}\left(\vartheta ; q^{-1}\right) \mathcal{R}\left(k ; q^{-1}\right) \quad-\right.$ $\left.\mathcal{B}\left(\vartheta ; q^{-1}\right) \mathcal{S}\left(k ; q^{-1}\right) q^{-1}\right\}, \vartheta \in \Theta_{k}$, are within the open circle with radius $\lambda<1$. We shall call $\lambda$ the stability margin.

For ease of notation, we shall call the controller with index $k \in \mathcal{K}$ simply as "controller $k$ ".

We can then define $\Sigma: \Theta \rightarrow \mathcal{K}$ to be the map associating the parameter $\vartheta$ with the controller $k$ which robustly stabilizes the models in the set $\Theta_{k}$ to which $\vartheta$ belongs (in the case when $\vartheta$ belongs to the frontier of two or more sets, $\vartheta$ is associated to the controller with the minimum index $k$ ).

\section{The hysteresis-based switching supervisor}

The supervisor orchestrating the switching among the candidate controllers is implemented as a hybrid system with input given by the $u$ and $y$ signals, and output given by the switching signal $\sigma$ taking values in $\mathcal{K}: \sigma_{t}$ is the index of the controller operating in closed-loop with the system at time $t$.

The operation of the supervisor can be described as follows. At each time $t>0$, the least squares (LS) cost:

$$
J_{t}(\vartheta)=\frac{1}{t+1} \sum_{s=0}^{t}\left(y_{s}-\varphi_{s-1}^{T} \vartheta\right)^{2}+\epsilon_{J}, \quad \epsilon_{J}>0
$$

is computed. Let $\hat{\vartheta}_{t}:=\arg \min \left\{J_{t}(\vartheta): \vartheta \in \Theta\right\}$. If

$$
(1+h) J_{t}\left(\hat{\vartheta}_{t}\right)<\min \left\{J_{t}(\vartheta): \vartheta \in \Theta_{\sigma_{t-1}}\right\},
$$

where $h>0$ is the hysteresis factor, then controller $\sigma_{t-1}$ is replaced by controller $\sigma_{t}=\Sigma\left(\hat{\vartheta}_{t}\right)$. The supervisor is hybrid because the switching logic is an event-driven system.

If we define the parameter estimate $\vartheta_{t}$ to be:

$\vartheta_{t}=\left\{\begin{array}{l}\hat{\vartheta}_{t}, \text { if }(1+h) J_{t}\left(\hat{\vartheta}_{t}\right)<\min \left\{J_{t}(\vartheta): \vartheta \in \Theta_{\Sigma\left(\vartheta_{t-1}\right)}\right\} \\ \vartheta_{t-1}, \text { otherwise, }\end{array}\right.$

initialized with $\vartheta_{-1}=\bar{\vartheta} \in \Theta$, then the hysteresis-based switching signal is simply given by

$$
\sigma_{t}=\Sigma\left(\vartheta_{t}\right)
$$

The idea underlying any estimator-based approach to switching is that, as the amount of data collected from the system increases, the estimated system with parameter $\hat{\vartheta}_{t}$ better resembles the behavior of the actual system, at least in closedloop (closed-loop identification property). Hence, by imposing a specific desired behavior on the estimated system, one actually imposes that behavior on the underlying system (selftuning property). The problem is that the parameter estimate is generally not consistent, and it may not even converge to any $\vartheta$ (see e.g. [13]). Hence, if the control law was continuously tuned to the parameter estimate $\hat{\vartheta}_{t}$, i.e., $\vartheta_{t}=\hat{\vartheta}_{t}$, for all $t$, then the "frozen" estimated system dynamics would be stabilized, but the stability of the time-varying estimated system will not be ensured. A possible solution to this issue is then to update the parameter estimate at a slower rate than the updating of the system variables, so as to limit the estimated system time variability, while preserving the closed-loop identification property of the adopted estimation method. In standard adaptive control, this is typically achieved through error normalization ([14]). In the hysteresis-based switching control, this is achieved by changing controller only at those times $t$ when the collected data reveal that the model used to select the currently operating controller is significantly worse than the best estimated model in terms of the value taken by the identification criterion $J_{t}$. If $J_{t}$ and $h$ are appropriately selected for the considered class of systems, this makes the switching slow on the average, hence securing stability of the time-varying estimated system. 


\section{Stability analysis}

The switching control system

$\left\{\begin{array}{l}y_{t+1}=\left[1-\mathcal{A}\left(\vartheta^{\circ} ; q^{-1}\right)\right] y_{t+1}+\mathcal{B}\left(\vartheta^{\circ} ; q^{-1}\right) u_{t}+w_{t+1} \\ u_{t}=\mathcal{S}\left(\sigma_{t} ; q^{-1}\right) y_{t}+\left[1-\mathcal{R}\left(\sigma_{t} ; q^{-1}\right)\right] u_{t},\end{array}\right.$

with $\sigma_{t}$ given by (4), can be represented as a variational system with respect to the closed-loop estimated system, i.e., the closed-loop system composed of the model with parameter $\vartheta_{t}$ controlled by the controller $\sigma_{t}$, as follows:

$$
\left\{\begin{array}{l}
y_{t+1}=\left[1-\mathcal{A}\left(\vartheta_{t} ; q^{-1}\right)\right] y_{t+1}+\mathcal{B}\left(\vartheta_{t} ; q^{-1}\right) u_{t}+w_{t+1}+e_{t} \\
u_{t}=\mathcal{S}\left(\sigma_{t} ; q^{-1}\right) y_{t}+\left[1-\mathcal{R}\left(\sigma_{t} ; q^{-1}\right)\right] u_{t}
\end{array}\right.
$$

where $e_{t}:=\varphi_{t}^{T}\left[\vartheta^{\circ}-\vartheta_{t}\right]$ is the estimation error.

The switching control system stability can then be proved based on the following two facts: i) by adopting the hysteresisbased switching logic based on the LS cost, uniform exponential stability of the closed-loop estimated system with $e_{t}$ regarded as an exogenous input is guaranteed; and ii) by switching from time to time to the controller designed for the best estimated model, the internally generated perturbation term $e_{t}$ is kept 'small'. These two properties are proved next.

\section{Uniform exponential stability}

Consider the closed-loop system

$$
\left\{\begin{array}{l}
\mathcal{A}\left(\vartheta ; q^{-1}\right) y_{t+1}=\mathcal{B}\left(\vartheta ; q^{-1}\right) u_{t} \\
\mathcal{R}\left(k ; q^{-1}\right) u_{t}=\mathcal{S}\left(k ; q^{-1}\right) y_{t}
\end{array}\right.
$$

By letting $x_{t}:=\left[y_{t} \ldots y_{t-n+1} u_{t-1} \ldots u_{t-m+1}\right]^{T}$ where $n:=$ $\max \left\{n_{s}, n_{c}+1\right\}$ and $m:=\max \left\{m_{s}, m_{c}+1\right\}$, system (6) can be given the state space representation

$$
\left\{\begin{array}{l}
x_{t+1}=A(\vartheta) x_{t}+B(\vartheta) u_{t} \\
u_{t}=L(k) x_{t}
\end{array}\right.
$$

where

$A(\vartheta)=\left[\begin{array}{cccc|cccc}a_{1} & \ldots & a_{n-1} & a_{n} & b_{2} & \ldots & b_{m-1} & b_{m} \\ 1 & 0 & \ldots & & 0 & \ldots & & 0 \\ & \ddots & \ddots & & & \ddots & & 0 \\ & & 1 & 0 & & & & 0 \\ \hline 0 & \ldots & \ldots & 0 & 0 & \ldots & \ldots & 0 \\ 0 & \ldots & \ldots & 0 & 1 & 0 & & \\ & \ddots & \ddots & & & \ddots & \ddots & \\ & & 0 & 0 & & & 1 & 0\end{array}\right]$

$B(\vartheta)=\left[b_{1} 0 \cdots 0 \mid 10 \cdots 0\right]$,

$L(k)=\left[s_{0}(k) \cdots s_{n-1}(k) s_{n}(k) \mid r_{1}(k) \cdots r_{m-1}(k) r_{m}(k)\right]$,

with $a_{i}=0$ if $i>n_{s}, s_{i}(k)=0$ if $i>n_{c}, b_{i}=0$ if $i>m_{s}$, $r_{i}(k)=0$ if $i>m_{c}$, thus leading to $x_{t+1}=F(\vartheta, k) x_{t}$, where

$$
F(\vartheta, k)=A(\vartheta)+B(\vartheta) L(k) .
$$

Note that the introduced state space representation of the model with parameter $\vartheta$ is nonminimal but, because of the block triangular matrix structure of $A(\vartheta)$, the added eigenvalues are all identically equal to zero. This, jointly with the fact that the stability margin is $\lambda$, implies that

$$
\max \left\{\left|\lambda_{\max }(F(\vartheta, \Sigma(\vartheta)))\right|: \vartheta \in \Theta\right\}<\lambda .
$$

Let us define the rescaled version of the performance index $J_{t}$ : $\bar{J}_{t}(\vartheta):=(t+1) J_{t}(\vartheta)$. It is easily seen that 1) $\bar{J}_{0}(\vartheta)>0, \vartheta \in$ $\Theta$, and 2) $\bar{J}_{t+1}(\vartheta) \geq \bar{J}_{t}(\vartheta), \forall t \geq 0, \vartheta \in \Theta$. Therefore, the scale-independent hysteresis switching theorem in [9] can be applied. Since such a theorem is in fact key for proving the uniform exponential stability of the closed-loop estimated system, we recall it below according to our notations.

Theorem 1 ([9]). Consider the switching control system (5) with switching signal $\sigma_{t}=\Sigma\left(\vartheta_{t}\right)$, where $\vartheta_{t}$ is defined in (3). Fix an arbitrary time interval $\left[t_{0}, t\right], 0 \leq t_{0}<t$, and denote with $N_{\sigma}\left(t_{0}, t\right)$ the number of switching times in $\left(t_{0}, t\right)$. Then,

$$
N_{\sigma}\left(t_{0}, t\right) \leq 1+m+\frac{m}{\log (1+h)} \log \frac{\bar{J}_{t}(\vartheta)}{\bar{J}_{t_{0}}\left(\hat{\vartheta}_{t_{0}}\right)}, \vartheta \in \Theta .
$$

Theorem 2. The autonomous system $x_{t+1}=F\left(\vartheta_{t}, \sigma_{t}\right) x_{t}$ with $\vartheta_{t}$ and $\sigma_{t}$ respectively given by (3) and (4) is a.s. exponentially stable, uniformly in time, i.e, there exists $\bar{\lambda} \in(\lambda, 1)$ such that, for all $t_{0}, t$, with $0 \leq t_{0} \leq t$,

$$
\left\|x_{t}\right\| \leq \bar{k} \bar{\lambda}^{t-t_{0}}\left\|x_{t_{0}}\right\|, \quad \text { a.s., }
$$

where $\bar{k}$ is a suitable random constant.

Proof. Given a square matrix $P$, we denote with $\mathcal{K}_{\#}(P)$ its condition number with respect to the 2-norm, which reduces to the ratio between its maximum and minimum eigenvalues if $P$ is symmetric. Set $\overline{\mathcal{K}}_{\#}:=\sup _{\vartheta \in \Theta} \mathcal{K}_{\#}(P(\vartheta))$, where $P(\vartheta)$ is the solution to the Lyapunov equation:

$$
\frac{1}{\lambda} F(\vartheta, \Sigma(\vartheta))^{T} P \frac{1}{\lambda} F(\vartheta, \Sigma(\vartheta))-P=-I .
$$

$\overline{\mathcal{K}}_{\#}$ is easily seen to be bounded. In the case when $\Theta$ is finite, this property immediately follows from the fact that, due to the stability margin property (8), $\frac{1}{\lambda} F(\vartheta, \Sigma(\vartheta)), \vartheta \in \Theta$, is stable, hence $P(\vartheta)$ is positive define and $\mathcal{K}_{\#}(P(\vartheta))<\infty$ for all $\vartheta \in$ $\Theta$. In the case when $\Theta=\cup_{k=1}^{m} \Theta_{k}$, where $\Theta_{k}$ is a continuum of parameterizations and it is compact, one has to use also the property that, for every $k \in \mathcal{K}, F(\vartheta, \Sigma(\vartheta))$ is a continuous function of $\vartheta, \vartheta \in \Theta_{k}$, hence $P(\vartheta)$ (and $\mathcal{K}_{\#}(P(\vartheta))$ ) is also continuous on $\Theta_{k}$ (see [15]).

The time-invariant system $v_{t+1}=\frac{1}{\lambda} F(\vartheta, \Sigma(\vartheta)) v_{t}$ is exponentially stable $\forall \vartheta \in \Theta$. Moreover, its Lyapunov function $\mathcal{V}_{t}(\vartheta):=v_{t}^{T} P(\vartheta) v_{t}$ satisfies

$$
\mathcal{V}_{t+1}(\vartheta)-\mathcal{V}_{t}(\vartheta)=-\left\|v_{t}\right\|^{2}
$$

Denote by $\left\{t_{i}\right\}$ the switching times sequence. Consider now the time-varying system $z_{t+1}=\frac{1}{\lambda} F\left(\vartheta_{t}, \Sigma\left(\vartheta_{t}\right)\right) z_{t}$. Since during each time interval $\left[t_{i}, t_{i+1}\right)$ this system is time invariant, by 
(11) we have $\mathcal{V}_{\bar{t}+1}\left(\vartheta_{t_{i}}\right) \leq \mathcal{V}_{t^{*}}\left(\vartheta_{t_{i}}\right), t^{*} \leq \bar{t}, t^{*}, \bar{t} \in\left[t_{i}, t_{i+1}\right)$. From this inequality we obtain the following bound

$$
\left\|z_{\bar{t}+1}\right\| \leq \rho\left\|z_{t^{*}}\right\|, \quad t^{*} \leq \bar{t}, t^{*}, \bar{t} \in\left[t_{i}, t_{i+1}\right),
$$

where $\rho:=\sqrt{\max _{\vartheta \in \Theta} \mathcal{K}_{\#}(P(\vartheta))}$. In particular, for $t^{*}=t_{i}$ and $\bar{t}=t_{i+1}-1$,

$$
\left\|z_{t_{i+1}}\right\| \leq \rho\left\|z_{t_{i}}\right\| .
$$

Consider now the time interval $\left[t_{0}, t\right]$.

Let $\left\{t_{k_{1}}, t_{k_{2}}, \ldots, t_{k_{N_{\sigma}\left(t_{0}, t\right)}}\right\}$, with $t_{0}<t_{k_{1}}<t_{k_{2}}<\ldots<$ $t_{k_{N_{\sigma}\left(t_{0}, t\right)}}<t$, denote the $N_{\sigma}\left(t_{0}, t\right)$ switching times in $\left(t_{0}, t\right)$. By applying first (12) with $t^{*}=t_{k_{N_{\sigma}\left(t_{0}, t\right)}}$ and $\bar{t}=t$, then, (13) repeatedly, and finally (12) with $t^{*}=t_{0}$ and $\bar{t}=t_{k_{1}}$, we obtain $\left\|z_{t}\right\| \leq \rho^{N_{\sigma}\left(t_{0}, t\right)+1}\left\|z_{t_{0}}\right\|$.

Setting $z_{t_{0}}=x_{t_{0}}$, we finally get a bound on the state vector $x_{t}$ of the time-varying system $x_{t+1}=F\left(\vartheta_{t}, \sigma_{t}\right) x_{t}$ :

$$
\left\|x_{t}\right\| \leq \rho^{N_{\sigma}\left(t_{0}, t\right)+1} \lambda^{t-t_{0}}\left\|x_{t_{0}}\right\| .
$$

We next derive a bound on $N_{\sigma}\left(t_{0}, t\right)$ based on Theorem 1 . By equation (9) with $\vartheta=\vartheta^{\circ}$, we have $N_{\sigma}\left(t_{0}, t\right) \leq$ $1+m+\frac{m}{\log (1+h)} \log \frac{(t+1)\left[\frac{1}{t+1} \sum_{s=0}^{t} w_{s}^{2}+\epsilon_{J}\right]}{\left(t_{0}+1\right) \epsilon_{J}}$. This inequality can be rewritten as follows: $N_{\sigma}\left(t_{0}, t\right) \leq 1+m+$ $\frac{m}{\log (1+h)}\left(\log \left(\frac{t+1}{t_{0}+1}\right)+\log \left(\frac{1}{\epsilon_{J}}\left[\frac{1}{t+1} \sum_{s=0}^{t} w_{s}^{2}+\epsilon_{J}\right]\right)\right)$.

From Assumption 1, we have that with probability 1 there exists a random time instant $t^{\prime}<\infty$ such that $\frac{1}{t+1} \sum_{s=0}^{t} w_{s}^{2} \leq$ $\sigma^{2}+\epsilon_{J}, t \geq t^{\prime}$. Therefore, $N_{\sigma}\left(t_{0}, t\right) \leq 1+m+$ $\frac{m}{\log (1+h)}\left(\log \left(\frac{t+1}{t_{0}+1}\right)+\log \left(\frac{1}{\epsilon_{J}}\left[\sigma^{2}+2 \epsilon_{J}\right]\right)\right), t \geq t^{\prime}$.

Now, observe that $\log \left(\frac{t+1}{t_{0}+1}\right)=\log \left(\frac{t-t_{0}}{t_{0}+1}+1\right) \leq \log \left(t-t_{0}+\right.$ $1)=o\left(t-t_{0}\right)$. This implies that, if we fix $\bar{\lambda} \in(\lambda, 1)$, there exists $\tau^{\prime}>0$ such that

$$
\log \left(\frac{t+1}{t_{0}+1}\right) \leq \frac{\log (1+h)}{m} \log _{\rho}\left(\frac{\bar{\lambda}}{\lambda}\right)\left(t-t_{0}\right),
$$

$t-t_{0} \geq \tau^{\prime}$ and therefore, $N_{\sigma}\left(t_{0}, t\right) \leq 1+m+$ $\frac{m}{\log (1+h)} \log \left(\frac{\sigma^{2}}{\epsilon_{J}}+2\right)+\log _{\rho}\left(\frac{\bar{\lambda}}{\lambda}\right)\left(t-t_{0}\right), t \geq t^{\prime}, t-t_{0} \geq \tau^{\prime}$.

By replacing this bound in equation (14), we have that $\left\|x_{t}\right\| \leq$ $\rho^{c_{1}+c_{2}\left(t-t_{0}\right)} \lambda^{t-t_{0}}\left\|x_{t_{0}}\right\|, t^{\prime} \leq t_{0} \leq t, t-t_{0} \geq \tau^{\prime}$, where $c_{1}:=$ $2+m+\frac{m}{\log (1+h)} \log \left(\frac{\sigma^{2}}{\epsilon_{J}}+2\right)$ and $c_{2}:=\log _{\rho}\left(\frac{\bar{\lambda}}{\lambda}\right)$. From this equation it then follows that $\left\|x_{t}\right\| \leq k \bar{\lambda}^{t-t_{0}}\left\|x_{t_{0}}\right\|, t^{\prime} \leq t_{0} \leq t$, $t-t_{0} \geq \tau^{\prime}$, where $k:=\rho^{c_{1}}$.

If $t-t_{0}<\tau^{\prime}$ or $0 \leq t_{0} \leq t<t^{\prime}$, then, $\left\|x_{t}\right\|$ can be bounded as follows

$$
\left\|x_{t}\right\| \leq\left(\max _{\vartheta \in \Theta}\|F(\vartheta, \Sigma(\vartheta))\|\right)^{t-t_{0}}\left\|x_{t_{0}}\right\| .
$$

If $0 \leq t_{0}<t^{\prime}<t$ and $t-t^{\prime} \geq \tau^{\prime}$,

$$
\left\|x_{t}\right\| \leq k \bar{\lambda}^{t-t^{\prime}}\left(\max _{\vartheta \in \Theta}\|F(\vartheta, \Sigma(\vartheta))\|\right)^{t^{\prime}-t_{0}}\left\|x_{t_{0}}\right\|,
$$

whereas if $0 \leq t_{0}<t^{\prime}<t$, but $t-t^{\prime}<\tau^{\prime}$, equation (15) holds. Observe now that by (14) with $t_{0}=$ $t-1$ and $x(t-1)=\bar{x},\|x(t)\|=\|F(\vartheta, \Sigma(\vartheta)) \bar{x}\| \leq$ $\rho \lambda\|\bar{x}\|, \bar{x} \in \Re^{n+m-1}, \quad \vartheta \in \Theta$. Since by definition $\|F(\vartheta, \Sigma(\vartheta))\|=\sup _{\|x\|=1}\|F(\vartheta, \Sigma(\vartheta)) x\|$, we have that $\max _{\vartheta \in \Theta}\|F(\vartheta, \Sigma(\vartheta))\|$ in equations (15) and (16) can be bounded as follows $\max _{\vartheta \in \Theta}\|F(\vartheta, \Sigma(\vartheta))\| \leq \rho \lambda$. Then, if we define the random constant $\bar{k}:=\max \left\{k \rho^{t^{\prime}}, \rho^{t^{\prime}+\tau^{\prime}}\right\}$ equation (10) is satisfied for all $t_{0}, t$ such that $0 \leq t_{0} \leq t$, which concludes the proof.

\section{Bound on the perturbation term}

Theorem 3. Suppose that $u_{t}$ is $\mathcal{F}_{t}$-measurable. Then, $\vartheta_{t}$ given in (3) satisfies

$\sum_{s=0}^{t}\left(\varphi_{s-1}^{T}\left(\vartheta^{\circ}-\vartheta_{t}\right)\right)^{2}=o\left(\sum_{s=0}^{t}\left\|\varphi_{s-1}\right\|^{2}\right)+h O(t+1), \quad$ a.s.

Proof. By definition (3), $\bar{J}_{t}\left(\vartheta_{t}\right) \leq(1+h) \bar{J}_{t}\left(\vartheta^{\circ}\right)$. This entails that $\sum_{s=0}^{t}\left(\varphi_{s-1}^{T}\left(\vartheta^{\circ}-\vartheta_{t}\right)\right)^{2} \leq h\left(\sum_{s=0}^{t} w_{s}^{2}+\right.$ $\left.(t+1) \epsilon_{J}\right)+2 \sum_{s=0}^{t} \varphi_{s-1}^{T}\left(\vartheta_{t}-\vartheta^{\circ}\right) w_{s} \leq h O(t+1)+$ $2 \sum_{s=0}^{t} \varphi_{s-1}^{T}\left(\vartheta_{t}-\vartheta^{\circ}\right) w_{s}$, where we used the fact that by Assumption $1, \sum_{s=0}^{t} w_{s}^{2}=O(t+1)$.

Observe now that $\varphi_{s-1}$ is $\mathcal{F}_{s-1}$-measurable whereas $w_{s}$ satisfies Assumption 1. Then, by Theorem 2.8 in [16] and Assumption $2, \sum_{s=0}^{t} \varphi_{s-1}^{T}\left(\vartheta_{t}-\vartheta^{\circ}\right) w_{s} \leq \max \left\{\left\|\vartheta_{1}-\vartheta_{2}\right\|: \vartheta_{1}, \vartheta_{2} \in\right.$ $\Theta\}\left\|\sum_{s=0}^{t} \varphi_{s-1}^{T} w_{s}\right\|=o\left(\sum_{s=0}^{t}\left\|\varphi_{s-1}\right\|^{2}\right)$, a.s., which concludes the proof.

The technical proof of the corollary below is obtained by a suitable manipulation of the sole result in Theorem 3 , jointly with the uniform boundedness of $\vartheta_{t}$. Its proof is similar to that of Proposition 3.3 in [10], hence is omitted due to space limitations (see [17]).

Corollary 1. Suppose that $u_{t}$ is $\mathcal{F}_{t}$-measurable. Then, the estimation error $e_{t}=\varphi_{t}^{T}\left(\vartheta^{\circ}-\vartheta_{t}\right)$ satisfies

$$
\sum_{t=0, t \notin \mathcal{B}_{N}}^{N} e_{t}^{2}=o\left(\sum_{t=0}^{N}\left\|\varphi_{t}\right\|^{2}\right)+h O(N+1), \quad \text { a.s. },
$$

where $\mathcal{B}_{N}$ is a set of instant points which depends on $N$, whose cardinality is bounded: $\left|\mathcal{B}_{N}\right| \leq C_{\mathcal{B}}, \forall N$.

\section{Stability result}

In Theorem 4 below, the switching control system (5) is shown to be stable. Theorem 4 follows from Theorem 2 and Corollary 1. Its proof is omitted due to space limitations. The interested reader is referred to [17].

Theorem 4. The switching control system (5) is $L^{2}$-stable:

$$
\limsup _{N \rightarrow \infty} \frac{1}{N} \sum_{t=0}^{N-1}\left[y_{t}^{2}+u_{t}^{2}\right]<\infty, \quad \text { a.s. }
$$


Remark 1. The hysteresis-based switching control method has been formulated here referring to the system description (1), which is linearly parameterized in the parameter vector $\vartheta^{\circ}$. This was done only for simplifying the presentation. The approach can in fact be easily reformulated to address the case when $\vartheta^{\circ}$ in (1) is a function of some $q$-dimensional parameter $p^{\circ}$ belonging to a certain set $\mathcal{P} \subset \Re^{q}$. If $\mathcal{P}$ is compact and $f: \mathcal{P} \rightarrow \Re^{n_{s}+m_{s}}$ is a continuous function on $\mathcal{P}$, then all the results proved for the linearly parameterized case remain valid.

\section{A simulation example}

In this section, we try to give a better insight into the hysteresisbased switching control approach by means of a simulation example. In particular, we analyze the influence of the hysteresis factor on the adaptation mechanism of the proposed hysteresisbased switching controller.

We consider a system described by ([3]):

$y_{t+1}=-p^{\circ} y_{t}+\frac{2}{9} p^{\circ}\left(p^{\circ}+2\right) y_{t-1}+u_{t}-\frac{1}{6}\left(p^{\circ}+2\right) u_{t-1}+w_{t+1}$, where $\left\{w_{t}\right\}$ is an i.i.d. Gaussian sequence with zero mean and variance $\sigma^{2}=10^{-4}$, and $p^{\circ}$ is a scalar parameter belonging to $\mathcal{P}:=[-3,3] \subset \Re$.

We partition the parameter set in five subsets $\mathcal{P}_{k}, k \in \mathcal{K}=$ $\{1,2, \ldots, 5\}$, as indicated in Figure 1 . We then associate with

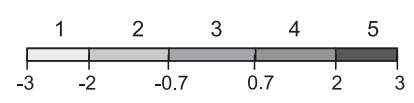

Figure 1: Partition of the uncertainty parameter set $\mathcal{P}$ in five subsets.

each $\mathcal{P}_{k}, k \in \mathcal{K}$, a controller of the form $\mathcal{R}\left(k ; q^{-1}\right) u_{t}=$ $\mathcal{S}\left(k ; q^{-1}\right) y_{t}, k \in \mathcal{K}$, where $\mathcal{R}\left(k ; q^{-1}\right)=1-r_{1}(k) q^{-1}$ and $\mathcal{S}\left(k ; q^{-1}\right)=s_{0}(k)+s_{1}(k) q^{-1}$, which robustly stabilizes all the models with parameter belonging to $\mathcal{P}_{k}$ with a certain stability margin $\lambda$. Precisely, we consider five controllers with coefficients vector $\gamma(k):=\left[s_{0}(k) s_{1}(k) r_{1}(k)\right]$, $k \in \mathcal{K}$, given by: $\gamma(1)=[-2.365-0.258-0.077], \gamma(2)=$ [-1.379 0.155 0.077], $\gamma(3)=[0.183-0.0870 .328], \gamma(4)=$ $[1.739-1.3060 .544]$, and $\gamma(5)=[2.724-2.4820 .677]$, and introduce the map $\Sigma: \mathcal{P} \rightarrow \mathcal{K}$ defined as: $\Sigma(p)=k$, if $p \in$ $\mathcal{P}_{k}$, which ensures a stability margin $\lambda=0.88$.

Let us denote by $\hat{p}_{t}$ the minimizer of the LS signal $J_{t}(p)$. Then, $\sigma_{t}$ is given by $\sigma_{t}=\Sigma\left(p_{t}\right)$, where

$p_{t}= \begin{cases}\hat{p}_{t}, & \text { if }(1+h) J_{t}\left(\hat{p}_{t}\right)<\min \left\{J_{t}(p): p \in \mathcal{P}_{\Sigma\left(p_{t-1}\right)}\right\} \\ p_{t-1}, & \text { otherwise. }\end{cases}$

All the simulations refer to the case when $p^{\circ}=-0.9, \epsilon_{J}=\sigma^{2}$, and the control system is initialized with $p_{-1}=0.8$ and $y_{-1}=y_{-2}=u_{-1}=0$. Moreover, the same realization of the noise process $\left\{w_{t}\right\}$ is used so as to better compare the control system behavior obtained for different choices of $h$. Yet, the conclusions drawn are valid in general, since the described simulation results are representative of the typical control system behavior.
Before presenting the simulation results, we make some remarks which will be useful for their interpretation.

The controller operating at $t-1$ is not replaced at time $t$ if and only if $\Delta J_{t}:=\min \left\{J_{t}(p): p \in \mathcal{P}_{\Sigma\left(p_{t-1}\right)}\right\}-J_{t}\left(\hat{p}_{t}\right)$ satisfies the condition

$$
\Delta J_{t} \leq h J_{t}\left(\hat{p}_{t}\right)
$$

In particular, no switching occurs at time $t$ if $\hat{p}_{t}$ belongs to the same set as $p_{t-1}$ since in this case, $\Delta J_{t}=0$. Also, the bound in (18) can be estimated as follows: $\Delta J_{t} \leq h J_{t}\left(\hat{p}_{t}\right) \leq h J_{t}\left(p^{\circ}\right)=$ $h\left(\frac{1}{t+1} \sum_{s=0}^{t} w_{s}^{2}+\epsilon_{J}\right), t \geq 0$, which, by Assumption 1 , tends to $h\left(\sigma^{2}+\epsilon_{J}\right)$, in the long run.

Figure 2 represents $\sigma_{t}$ in the cases when $h=0.01, h=0.1$, $h=1$, and $h=10$. Since after $t=50, \sigma_{t}$ remains constant in our simulations, we report only the time interval $[0,50]$.

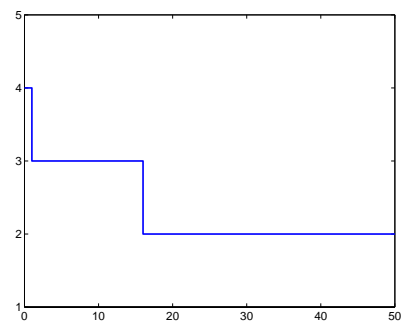

(a)

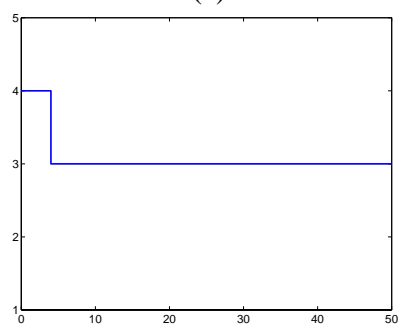

(c)

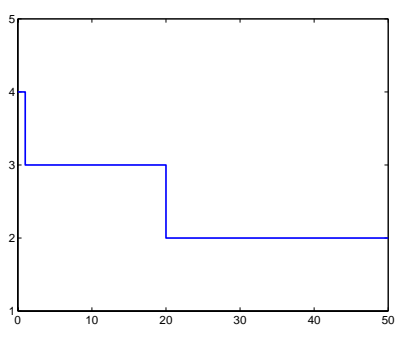

(b)

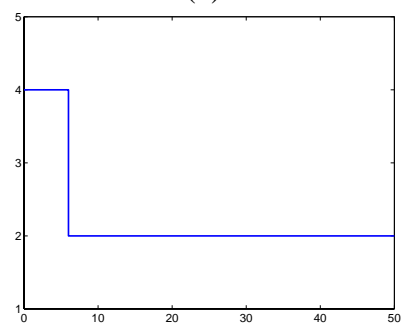

(d)
Figure 2: $\sigma_{t}, t \in[0,50]$, for (a) $h=0.01$; (b) $h=0.1$; (c) $h=1$; and (d) $h=10$.

At time $t=0$, the hysteresis-based switching logic places in feedback with the system the controller $k=\Sigma\left(p_{0}\right)$. Here, $\Sigma\left(p_{0}\right)=\Sigma\left(p_{-1}\right)=4$, and this irrespectively of the value of the hysteresis factor $h$.

For small $h(h=0.01$ and $h=0.1)$, the positive value taken by $\Delta J_{1}$ violates condition (18), thus causing controller 4 introduced at $t=0$ to be replaced by controller 3 at time 1 . By increasing the value of $h$, one increases the response time of the adaptation mechanism. In our example, when $h=1$ and $h=10$, condition (18) is satisfied at $t=1$, hence controller 4 is kept in the loop. However, eventually controller 4 will be certainly changed, since it destabilizes the true system. The more controller 4 is kept in the loop, the more the system dynamics is excited, thus possibly causing the LS estimate $\hat{p}_{t}$ to rapidly converge to the true parameter $p^{\circ}$. This is actually what happens for $h=10$. The switching logic with $h=10$ decides to switch to a different controller after 6 time instants. Since $\hat{p}_{t}$ is already close to $p^{\circ}$ at $t=6$, controller 2 is placed 
in the loop at $t=6$ and then it is no more falsified. As for $h=1$, the excitation introduced in the initial (shorter) phase when controller 4 is operating in the loop is not sufficient for $\hat{p}_{t}$ to get close to $p^{\circ}$ at the switching time. Controller 4 is in fact replaced by controller 3 . Moreover, an interesting fact is that controller 3 is then kept in the loop forever. This is because controller 3 stabilizes the true system, though $p^{\circ}$ has been associated with controller 2 (see Figure 3). Therefore, $\Delta J_{t}$ keeps bounded when controller 3 is maintained in the loop. As it is seen in Figure 4, in the case when $h=1, \Delta J_{t}$ not only remains bounded, but it also keeps below $2 h\left(\sigma^{2}+\epsilon_{J}\right)=210^{-4}$ which is the estimated bound on the right-hand-side of equation (18). This is not the case when $h=0.1$ and $h=0.01$. For these values of $h$ the bound $2 h 10^{-4}$ is in fact overcome at some point, and controller 3 is falsified and replaced by controller 2 (cf. (a) and (b) in Figure 2).

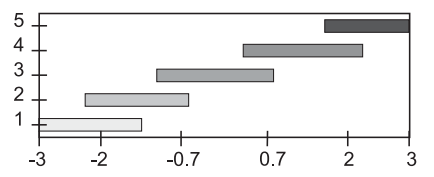

Figure 3: Stability regions associated with the candidate controllers.

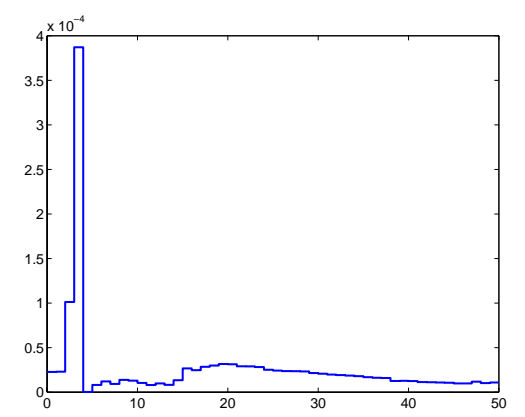

Figure 4: $\Delta J_{t}, t \in[0,50]$, when $h=1$.

\section{Concluding remarks}

We studied the hysteresis switching control solution to the problem of adaptively controlling an uncertain discrete-time linear system affected by a (possibly unbounded) stochastic noise.

The advantage of a hysteresis-based solution over a dwell-time one is that in the former switching times are dictated by the response of the controlled system to the applied input, and not predefined. This is useful during transients, while the process uncertainty is still large.

We focused on ARX process models subject to white noise. The extension to ARMAX models is a possible avenue for future research.

\section{Acknowledgments}

Research supported by the European Commission under the project HYBRIDGE IST-2001-32460, by MIUR under the project "New techniques for the identification and adaptive control of industrial systems", and by the National Science Foundation under the grant ECS-0242798.

\section{References}

[1] R.H. Middleton, G.C. Goodwin, D.J. Hill, and D.Q. Mayne. Design issues in adaptive control. IEEE Trans. on Automatic Control, AC-33:50-58, 1988.

[2] A.S. Morse. Control using logic-based switching. In A. Isidori, editor, Trends in Control, pages 69-113. Springer-Verlag, New York, 1995.

[3] A.S. Morse. Supervisory control of families of linear set-point controllesr-Part 1: Exact matching. IEEE Trans. on Automatic Control, AC-41:1413-1431, 1996.

[4] A.S. Morse. Supervisory control of families of linear set-point controllesr-Part 2: Robustness. IEEE Trans. on Automatic Control, AC-42:1500-1515, 1997.

[5] J.P. Hespanha. Logic-based switching algorithms in control. $\mathrm{PhD}$ thesis, Dept. of Electrical Engineering, Yale University, 1998.

[6] F.M. Pait and F. Kassab Jr. On a class of switched, robustly stable, adaptive systems. In Proc. 38th Conf. on Decision and Control, pages 2673-2678, December 1999.

[7] D. Borrelli, A.S. Morse, and E. Mosca. Discrete-time supervisory control of families of 2-DOF linear set-point controllers. IEEE Trans. on Automatic Control, AC-44:178-181, 1999.

[8] B. D. O. Anderson, T. S. Brinsmead, F. de Bruyne, J. Hespanha, D. Liberzon, and A. S. Morse. Multiple model adaptive control. I: Finite controller coverings. Int. J. of Robust and Nonlinear Control, 10:909-929, 2000. George Zames Special Issue.

[9] J.P. Hespanha, D. Liberzon, and A. S. Morse. Bounds on the number of switchings with scale-independent hysteresis: Applications to supervisory control. In Proc. 39th Conf. on Decision and Control, December 2000.

[10] M. Prandini and M.C. Campi. Adaptive LQG control of inputoutput systems - A cost-biased approach. SIAM J. Control and Optim., 39(5):1499-1519, 2001.

[11] M. Prandini and M.C. Campi. Logic-based switching for the stabilization of stochastic systems in presence of unmodeled dynamics. In Proc. 40th Conf. on Decision and Control, Orlando, FL, USA, December 2001.

[12] J. Hespanha, D. Liberzon, A. S. Morse, B.D.O. Anderson, T.S. Brinsmead, and F. de Bruyne. Multiple model adaptive control. II: Switching. Int. J. of Robust and Nonlinear Control, 11(5):479-496, 2001.

[13] K. Nassiri-Toussi and W. Ren. On the convergence of least squares estimates in white noise. IEEE Trans. on Automatic Control, AC-39:364-368, 1994.

[14] G.C. Goodwin and K.S. Sin. Adaptive filtering prediction and control. Englewood Cliffs, NJ: Prentice-Hall, 1984.

[15] D.F. Delchamps. Analytic feedback control and the algebraic Riccati equation. IEEE Trans. on Automatic Control, AC29:1031-1033, 1984.

[16] H.F. Chen and L. Guo. Identification and Stochastic Adaptive Control. Birkhäuser, Boston, 1991.

[17] M. Prandini, J.P. Hespanha, and M.C. Campi. Hysteresis-based switching control of stochastic linear systems. Technical report, University of Brescia, Brescia, Italy, April 2002. 